\title{
A computer program to determine the soil textural class in 1-2-3 for Windows and
}

\section{EXCEL}

\begin{abstract}
Spreadsheets are now widely used for data entry and analysis. Therefore, Texture AutoLookup (TAL) is a computer program designed to work within 1ï 2 ï 3 for Windows and EXCEL to determine the USDA soil textural class. TAL determines the textural class without having to repeat data entry because data is taken directly from the spreadsheet itself. Moreover, TAL works even with two particle size data or with imperfect data (that is, the sum of the three particle sizes being unequal to 100\%). TAL is independent of the particlesize analysis method, and TAL allows textural class names to be modified or be translated into another language.
\end{abstract}

Keyword: Soil textural class; Texture AutoLookup (TAL) 